\title{
DYNAMIC BEHAVIORS OF AN AMENSALISM SYSTEM WITH DENSITY DEPENDENT BIRTH RATE
}

\author{
YIQIN WANG \\ Department of Mathematics Research, Fujian Institute of Education, Fuzhou 350001, Fujian, China
}

\begin{abstract}
In this paper, an amensalism system with density dependent birth rate is proposed and studied The system admits four nonnegative equilibria. We show that all of the four equilibria are globally attractive under some suitable assumption. The birth rate of species plays an important role on the dynamic behaviors of the system.
\end{abstract}

Keywords. Amensalism system; Density dependent birth rate; Global attractivity.

2010 Mathematics Subject Classification. 34C25, 92D25.

\section{INTRODUCTION}

The aim of this paper is to investigate the dynamic behaviors of the following amensalism system with density dependent birth rate

$$
\begin{aligned}
& \frac{d x}{d t}=x\left(\frac{b_{11}}{b_{12}+x}-b_{13}-a_{11} x-a_{12} y\right) \\
& \frac{d y}{d t}=y\left(\frac{b_{21}}{b_{22}+y}-b_{23}-a_{22} y\right)
\end{aligned}
$$

where $b_{i j}, i=1,2, j=1,2,3, a_{11}, a_{12}$ and $a_{22}$ are all positive constants, and $x(t), y(t)$ are the density of the first and second species at time $t$, respectively. Here, we make the following assumptions:

(1) $\frac{b_{11}}{b_{12}+x}$ is the birth rate of the first species, which is density dependent;

(2) $b_{13}$ is the death rate of the first species, and $a_{11}$ is the density dependent coefficients of the first species;

(3) $\frac{b_{21}}{b_{22}+y}$ is the birth rate of the second species;

(4) $b_{23}$ is the death rate of the second species, and $a_{22}$ is the density dependent coefficients of the second species;

(5) The relationship between two species is amensalism.

E-mail address: 13905927851@126.com.

Received June 24, 2018; Accepted August 23, 2018.

(C)2018 Journal of Nonlinear Functional Analysis 
Amensalism, which means that a species inflicts harm to the other species without any costs or benefits received by the other, has recently been studied by many scholars; see $[1,2,3,4,5,6,7,8,9,10,11$, $12,13]$ and the references therein. The stability of the equilibrium $[7,8,9,10,11]$, the existence of the positive periodic solution [6], the extinction of the species [5], the influence of the cover [11, 12], and the influence of the functional response [13] have been extensively studied.

In [7], Zhu and Chen studied the following more general amensalism model

$$
\begin{aligned}
\frac{d x_{1}}{d t} & =x_{1}\left(b_{1}-a_{11} x_{1}-a_{12} x_{2}\right), \\
\frac{d x_{2}}{d t} & =x_{2}\left(b_{2}-a_{22} x_{2}\right) .
\end{aligned}
$$

They showed that if $b_{1} a_{22}>b_{2} a_{12}$, then system (1.2) admits a unique positive equilibrium $\left(x_{1}^{*}, x_{2}^{*}\right)$, which is globally attractive. If $b_{1} a_{22}<b_{2} a_{12}$, then the boundary equilibrium $\left(0, \frac{b_{2}}{a_{22}}\right)$ is globally attractive.

In [10], Chen proposed the following non-selective harvesting Lotka-Volterra amensalism model incorporating partial closure for the populations:

$$
\begin{aligned}
\frac{d N_{1}}{d t} & =r_{1} N_{1}\left(1-\frac{N_{1}}{P_{1}}-u \frac{N_{2}}{P_{1}}\right)-q_{1} E m N_{1}, \\
\frac{d N_{2}}{d t} & =r_{2} N_{2}\left(1-\frac{N_{2}}{P_{2}}\right)-q_{2} E m N_{2},
\end{aligned}
$$

where $u, r_{i}, P_{i}, i=1,2$ are all positive constants, $r_{i}\left(P_{i}\right)$ represents the intrinsic growth rate (environmental carrying capacity) of the $i$-th species, $E$ is the combined fishing effort used to harvest and $m(0<m<1)$ is the fraction of the stock available for harvesting. Chen showed that, based on the fraction of the stock available for harvesting, the system maybe extinctive, partial survival or two species coexist in a stable state. $\mathrm{Wu}[9]$ proposed the following nonlinear amensalism model

$$
\begin{aligned}
\frac{d N_{1}}{d t} & =r_{1} N_{1}\left(1-\left(\frac{N_{1}}{P_{1}}\right)^{\alpha_{1}}-u\left(\frac{N_{2}}{P_{1}}\right)^{\alpha_{2}}\right), \\
\frac{d N_{2}}{d t} & =r_{2} N_{2}\left(1-\left(\frac{N_{2}}{P_{2}}\right)^{\alpha_{3}}\right),
\end{aligned}
$$

where $u, r_{i}, P_{i}, i=1,2, \alpha_{j}, j=1,2,3$ are all positive constants. By using the differential inequality theory, $\mathrm{Wu}$ investigated the stability property of the equilibria of system (1.4). In [11], Xie, Chen and He considered a two species amensalism model with a partial cover for the first species to protect it from the second species. The model is as follows

$$
\begin{aligned}
& \frac{d x}{d t}=a_{1} x(t)-b_{1} x^{2}(t)-c_{1}(1-k) x(t) y(t), \\
& \frac{d y}{d t}=a_{2} y(t)-b_{2} y^{2}(t),
\end{aligned}
$$

where $a_{i}, b_{i}, i=1,2$ and $c_{1}$ are all positive constants, $k$ is a cover provided for the species $x$, and $0<k<1$. They showed that the conditions which ensure the local stability of $E_{2}\left(0, \frac{a_{2}}{b_{2}}\right)$ is enough to ensure its global stability. The positive equilibrium exists. And it is globally stable. In [12], Wu, Zhao and Lin further proposed a two species amensalism model with Holling II functional response and a partial cover for the first species. The author showed that the dynamic behaviors of the system is similar to that of system (1.4). Wu [13] also studied the dynamic behaviors of a two species amensalism model with nonmonotonic functional response. It bring to our attention that, in the above systems, they are assumed that 
the intrinsic growth rate of the species are constants, for example, if we do not consider the relationship between the two species, then, the equation of the first species, in system (1.3), takes the form

$$
\frac{d x}{d t}=x\left(b_{1}-a_{11} x\right)
$$

where $b_{1}$ is the intrinsic growth rate, and $a_{11}$ is the density dependent coefficients. System (1.6) could be revised as

$$
\frac{d x}{d t}=x\left(b_{11}-b_{13}-a_{11} x\right)
$$

where $b_{11}$ is the birth rate of the species and $b_{13}$ is the death rate of the species. Brauer and CastilloChavez [14], Tang and Chen [15], Berezansky, Braverman and Idels [16] showed that, in some cases, the density dependent birth rate of the species is more suitable. If we take the famous Beverton-Holt function [16] as the birth rate, then system (1.7) should be revised to

$$
\frac{d x}{d t}=x\left(\frac{b_{11}}{b_{12}+x}-b_{13}-a_{11} x\right) .
$$

Similarly, the second species could be expressed as follow

$$
\frac{d y}{d t}=y\left(\frac{b_{21}}{b_{22}+y}-b_{23}-a_{22} y\right) \text {. }
$$

In the study of the dynamic behaviors of the ecosystem, the stability, persistent and extinction are the most important topics, see $[3,4,15,16]$ and the references cited therein. For system (1.1), it is natural to investigate the extinct and stability property of the system. Also, it is natural to find out the influence of the density dependent birth rate. The rest of the paper is organized as follows. In Section 2, we investigate the stability property of the equilibria of system (1.1). In Section 3, we present some numerical simulations to show the feasibility of the main results. Finally, we end this paper by a briefly discussion.

\section{Global Stability}

The following Lemma, which is due to Chen et al. [17], plays an important role in this paper.

Lemma 2.1. Consider the following equation

$$
\frac{d y}{d t}=y\left(\frac{a}{b+y}-d-e y\right) \text {. }
$$

If $a>b d$, then the unique positive equilibrium $y^{*}$ of system (2.1) is globally asymptotically stable.

Now, we are in a position to consider the existence and stability property of the equilibria of system (1.1). If

$$
\frac{b_{21}}{b_{22}}>b_{23}
$$

then

$$
\frac{b_{21}}{b_{22}+y}-b_{23}-a_{22} y=0
$$

admits a unique positive solution $y^{*}$, where

$$
y^{*}=\frac{-\left(b_{23}+a_{22} b_{22}\right)+\sqrt{\left(b_{23}+a_{22} b_{22}\right)^{2}-4 a_{22}\left(b_{23} b_{22}-b_{21}\right)}}{2 a_{22}} .
$$

If

$$
\frac{b_{11}}{b_{12}}>b_{13}
$$


then

$$
\frac{b_{11}}{b_{12}+x}-b_{13}-a_{11} x=0
$$

admits a unique positive solution $x^{*}$, where

$$
x^{*}=\frac{-\left(b_{13}+a_{11} b_{12}\right)+\sqrt{\left(b_{13}+a_{11} b_{12}\right)^{2}-4 a_{11}\left(b_{13} b_{12}-b_{11}\right)}}{2 a_{11}} .
$$

If

$$
\frac{b_{11}}{b_{12}}>b_{13}+a_{12} y^{*}
$$

then

$$
\frac{b_{11}}{b_{12}+x}-b_{13}-a_{11} x-a_{12} y^{*}=0 .
$$

admits a unique positive solution

$$
x^{* *}=\frac{-\left(\left(b_{13}+a_{12} y^{*}\right)+a_{11} b_{12}\right)+\sqrt{\Delta}}{2 a_{11}},
$$

where

$$
\Delta=\left(\left(b_{13}+a_{12} y^{*}\right)+a_{11} b_{12}\right)^{2}-4 a_{11}\left(\left(b_{13}+a_{12} y^{*}\right) b_{12}-b_{11}\right) .
$$

The equilibrium of system (1.1) is determined by the equation

$$
\begin{aligned}
x\left(\frac{b_{11}}{b_{12}+x}-b_{13}-a_{11} x-a_{12} y\right) & =0, \\
y\left(\frac{b_{21}}{b_{22}+y}-b_{23}-a_{22} y\right) & =0 .
\end{aligned}
$$

The system always admits a boundary equilibrium $A_{1}(0,0)$. If (2.5) and

$$
\frac{b_{21}}{b_{22}}<b_{23}
$$

hold, then system (1.1) admits the nonnegative boundary equilibrium $A_{2}\left(x^{*}, 0\right)$, where $x^{*}$ is defined by (2.7). If (2.2) and

$$
\frac{b_{11}}{b_{12}}<b_{13}+a_{12} y^{*}
$$

hold, then system (1.1) admits the nonnegative boundary equilibrium $A_{3}\left(0, y^{*}\right)$. Assume that (2.2) and (2.8) holds. Then system (1.1) admits the unique positive equilibrium $A_{4}\left(x^{* *}, y^{*}\right)$, where $x^{* *}$ is defined by (2.10) and $y^{*}$ is defined by (2.4). In view of the stability property of the above four nonnegative equilibria, we have the following result.

Theorem 2.2. (1) If (2.2) and (2.8) holds, then system (1.1) admits a unique positive equilibrium $A_{4}\left(x^{* *}, y^{*}\right)$, which is globally asymptotically stable;

(2) If (2.2) and (2.14) holds, then the boundary equilibrium $A_{3}\left(0, y^{*}\right)$ is globally attractive;

(3) (2.5) and (2.13) holds, then the boundary equilibrium $A_{2}\left(x^{*}, 0\right)$ is globally attractive;

(4) If

$$
b_{11}<b_{12} b_{13}
$$

and (2.13) holds, then the boundary equilibrium $A_{1}(0,0)$ is globally attractive. 
Proof. (1) It is obvious that $x^{* *}$ and $y^{*}$ satisfy the equations

$$
\begin{array}{r}
\frac{b_{11}}{b_{12}+x^{* *}}-b_{13}-a_{11} x^{* *}-a_{12} y^{*}=0, \\
\frac{b_{21}}{b_{22}+y^{*}}-b_{23}-a_{22} y^{*}=0 .
\end{array}
$$

Consider the Lyapunov function

$$
V_{1}(x, y)=\frac{a_{11} a_{22}}{a_{12}^{2}}\left(x-x^{* *}-x^{* *} \ln \frac{x}{x^{* *}}\right)+\frac{1}{4}\left(y-y^{*}-y^{*} \ln \frac{y}{y^{*}}\right) .
$$

It is easy to see that $V_{1}$ is zero at the positive equilibrium $A_{4}\left(x^{* *}, y^{*}\right)$ and is positive for all other positive values of $x, y$. Applying (2.16), the time derivative of $V_{1}$ along the trajectories of (1.1) is

$$
\begin{aligned}
D^{+} V_{1}(t)= & \frac{a_{11} a_{22}}{a_{12}^{2}}\left(x-x^{* *}\right)\left(\frac{b_{11}}{b_{12}+x}-\frac{b_{11}}{b_{12}+x^{* *}}\right. \\
& \left.+a_{11} x^{* *}+a_{12} y^{*}-a_{11} x-a_{12} y\right) \\
& +\frac{1}{4}\left(y-y^{*}\right)\left(\frac{b_{21}}{b_{22}+y}-\frac{b_{21}}{b_{22}+y^{*}}+a_{22} y^{*}-a_{22} y\right) \\
= & -\frac{a_{11} a_{22}}{a_{12}^{2}} \frac{b_{11}}{\left(b_{12}+x\right)\left(b_{12}+x^{* *}\right)}\left(x-x^{* *}\right)^{2} \\
& -\frac{a_{11}^{2} a_{22}}{a_{12}^{2}}\left(x-x^{* *}\right)^{2}-\frac{a_{11} a_{22}}{a_{12}}\left(x-x^{* *}\right)\left(y-y^{*}\right) \\
& -\frac{1}{4} \frac{b_{21}}{\left(b_{22}+y\right)\left(b_{22}+y^{*}\right)}\left(y-y^{*}\right)^{2}-\frac{1}{4} a_{22}\left(y-y^{*}\right)^{2} \\
= & -\frac{a_{11} a_{22}}{a_{12}^{2}} \frac{b_{11}}{\left(b_{12}+x\right)\left(b_{12}+x^{* *}\right)}\left(x-x^{* *}\right)^{2} \\
& -\frac{1}{4} \frac{b_{21}}{\left(b_{22}+y\right)\left(b_{22}+y^{*}\right)}\left(y-y^{*}\right)^{2} \\
& -a_{22}\left[\frac{a_{11}}{a_{12}}\left(x-x^{* *}\right)+\frac{1}{2}\left(y-y^{*}\right)\right]^{2} .
\end{aligned}
$$

It then follows from (2) that $D^{+} V_{1}(t)<0$ strictly for all $x, y>0$ except the positive equilibrium $A_{4}\left(x^{* *}, y^{*}\right)$, where $D^{+} V_{1}(t)=0$. Thus, $V_{1}(x, y)$ satisfies Lyapunov's asymptotic stability theorem [18], and the positive equilibrium $A_{4}\left(x^{* *}, y^{*}\right)$ of system (1.1) is globally asymptotically stable.

(2) Inequality (2.14) implies that, for enough small positive constant $\varepsilon$,

$$
\frac{b_{11}}{b_{12}}<b_{13}+a_{12}\left(y^{*}-\varepsilon\right) .
$$

It follows from Lemma 2.1 and (2.2) that the system

$$
\frac{d y}{d t}=y\left(\frac{b_{21}}{b_{22}+y}-b_{23}-a_{22} y\right)
$$

admits a unique positive equilibrium $y^{*}$, which is globally asymptotically stable, that is,

$$
\lim _{t \rightarrow+\infty} y(t)=y^{*} .
$$

Hence, for $\varepsilon$ satisfies (2.19), there exists an enough large $T_{1}>0$ such that

$$
y^{*}-\varepsilon<y(t)<y^{*}+\varepsilon \text { for all } t \geq T_{1} .
$$


For $t>T_{1}$, it follows from (2.22) and the first equation of (1.1) that

$$
\begin{aligned}
\frac{d x}{d t} & \leq x\left(\frac{b_{11}}{b_{12}+x}-b_{13}-a_{11} x-a_{12}\left(y^{*}-\varepsilon\right)\right) \\
& \leq x\left(\frac{b_{11}}{b_{12}}-b_{13}-a_{12}\left(y^{*}-\varepsilon\right)\right) .
\end{aligned}
$$

Hence,

$$
x(t) \leq x\left(T_{1}\right) \exp \left\{\left(\frac{b_{11}}{b_{12}}-b_{13}-a_{12}\left(y^{*}-\varepsilon\right)\right)\left(t-T_{1}\right)\right\} \rightarrow 0 \text { as } t \rightarrow 0 .
$$

(3) For $\varepsilon>0$ enough small, it follows from (2.5) that

$$
\frac{b_{11}}{b_{12}}>b_{13}+a_{12} \varepsilon \text {. }
$$

Therefore,

$$
\begin{aligned}
\frac{d y}{d t} & =y\left(\frac{b_{21}}{b_{22}+y}-b_{23}-a_{22} y\right) \\
& \leq y\left(\frac{b_{21}}{b_{22}}-b_{23}\right)
\end{aligned}
$$

Hence,

$$
y(t) \leq y(0) \exp \left\{\left(\frac{b_{21}}{b_{22}}-b_{23}\right) t\right\} \rightarrow 0 \text { as } t \rightarrow 0 .
$$

Therefore, for $\varepsilon>0$ enough small, there exists an enough large $T_{2}>0$, such that

$$
y(t)<\varepsilon \text { for all } t \geq T_{2} .
$$

For $t>T_{2}$, it follows from the first equation of (1.1) that

$$
\frac{d x}{d t} \leq x\left(\frac{b_{11}}{b_{12}+x}-b_{13}-a_{11} x\right) .
$$

Consider the equation

$$
\frac{d u}{d t}=u\left(\frac{b_{11}}{b_{12}+u}-b_{13}-a_{11} u\right) .
$$

From (2.5) and Lemma 2.1, we see that system (2.30) admits a unique positive equilibrium $x^{*}$, which is globally asymptotically stable. From (2.29) and the differential inequality theory, one has

$$
\limsup _{t \rightarrow+\infty} x(t) \leq x^{*}
$$

For $t>T_{2}$, we also have

$$
\frac{d x}{d t} \geq x\left(\frac{b_{11}}{b_{12}+x}-b_{13}-a_{11} x-a_{12} \varepsilon\right) .
$$

Consider the equation

$$
\frac{d v}{d t}=v\left(\frac{b_{11}}{b_{12}+v}-b_{13}-a_{11} v-a_{12} \varepsilon\right) .
$$

Using Lemma 2.1, we see that system (2.33) admits a unique positive equilibrium $v^{*}(\varepsilon)$, which is globally asymptotically stable, where

$$
v^{*}(\varepsilon)=\frac{-\left(\left(b_{13}+a_{12} \varepsilon\right)+a_{11} b_{12}\right)+\sqrt{\Delta_{1}}}{2 a_{11}}
$$

and

$$
\Delta_{1}=\left(\left(b_{13}+a_{12} \varepsilon\right)+a_{11} b_{12}\right)^{2}-4 a_{11}\left(\left(b_{13}+a_{12} \varepsilon\right) b_{12}-b_{11}\right) .
$$


From (2.32) and the differential inequality theory, one has

$$
\liminf _{t \rightarrow+\infty} x(t) \geq v^{*}(\varepsilon) .
$$

It follows that

$$
v^{*}(\varepsilon) \leq \liminf _{t \rightarrow+\infty} x(t) \leq \limsup _{t \rightarrow+\infty} x(t) \leq x^{*} .
$$

Notethat $\varepsilon$ is enough small positive constant, and $v^{*}(\varepsilon) \rightarrow x^{*}$ as $\varepsilon \rightarrow 0$. Setting $\varepsilon \rightarrow 0$ in (2.36) leads to

$$
\lim _{t \rightarrow+\infty} x(t)=x^{*}
$$

(4) Under the assumption (2.13), we can find that

$$
y(t) \rightarrow 0 \text { as } t \rightarrow 0 .
$$

From the first equation of (1.1) and (2.15), we also have

$$
\begin{aligned}
\frac{d x}{d t} & \leq x\left(\frac{b_{11}}{b_{12}+x}-b_{13}-a_{11} x\right) \\
& \leq x\left(\frac{b_{11}}{b_{12}}-b_{13}\right) .
\end{aligned}
$$

Hence

$$
x(t) \leq x(0) \exp \left\{\left(\frac{b_{11}}{b_{12}}-b_{13}\right) t\right\} \text { as } t \rightarrow 0 .
$$

This ends the proof of Theorem 2.2.

\section{NUMERIC SIMULATIONS}

In this section, we consider the following four examples.

\section{Example 3.1.}

$$
\begin{aligned}
& \frac{d x}{d t}=x\left(\frac{1}{1+x}-2-x-5 y\right), \\
& \frac{d y}{d t}=y\left(\frac{1}{1+y}-2-2 y\right) .
\end{aligned}
$$

In this system, corresponding to system (1.1), we take $b_{11}=b_{12}=a_{11}=b_{21}=b_{22}=1, b_{13}=b_{23}=2$, $a_{22}=2, a_{12}=5$. Since $b_{11}<b_{12} b_{13}$ and $b_{21}<b_{22} b_{23}$, it follows from Theorem 2.2 (4) that boundary equilibrium $A_{1}(0,0)$ is globally asymptotically stable.

\section{Example 3.2.}

$$
\begin{aligned}
& \frac{d x}{d t}=x\left(\frac{4}{1+x}-2-x-5 y\right), \\
& \frac{d y}{d t}=y\left(\frac{1}{1+y}-2-2 y\right) .
\end{aligned}
$$

In this system, corresponding to system (1.1), we take $b_{11}=4, b_{12}=a_{11}=b_{21}=b_{22}=1, b_{13}=b_{23}=2$, $a_{22}=2, a_{12}=5$. Since $b_{11}>b_{12} b_{13}$ and $b_{21}<b_{22} b_{23}$, it follows from Theorem 2.2 (3) that boundary equilibrium $A_{2}(0.5615,0)$ is globally asymptotically stable. 


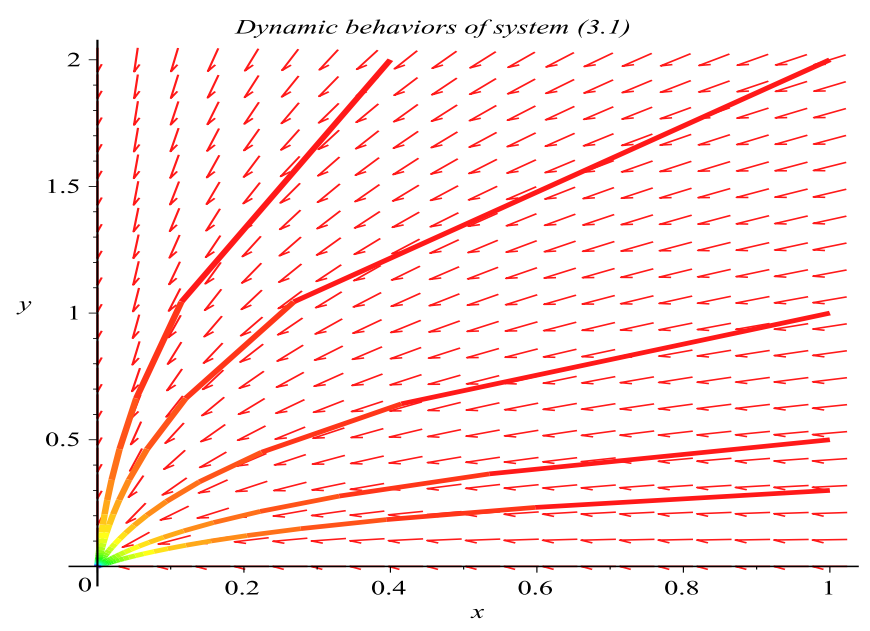

FIGURE 1. Dynamic behaviors of system (3.1) with the initial condition $(x(0), y(0))=(1,0.3),(0.4,2),(1,1),(1,2)$ and $(1,0.5)$, respectively.

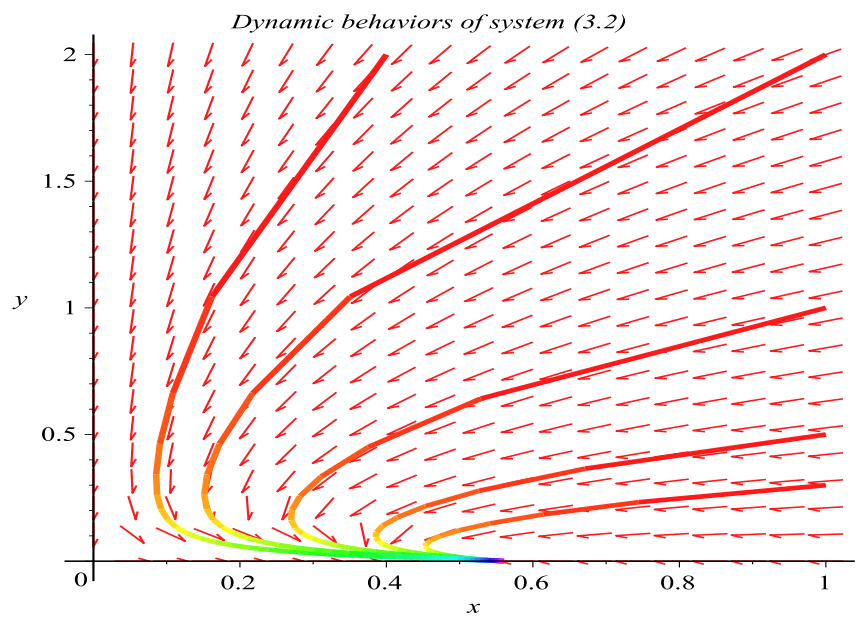

FIGURE 2. Dynamic behaviors of system (3.2) with the initial condition $(x(0), y(0))=(1,0.3),(0.4,2),(1,1),(1,2)$ and $(1,0.5)$, respectively.

\section{DisCUSSION}

Recently, many scholars have studied the dynamic behaviors of the ammensalism model. All these results are based on the traditional Logistic model. Specially, Zhu and Chen [7] showed that system (1.2) admits a unique positive equilibrium which is globally asymptotically stable or the first species will be driven to extinction while the second species is globally asymptotically stable to its steady-state. In this paper, we showed that the birth rate of the species maybe density dependent, and proposed the system (1.1). In Theorem 2.2, we showed that, under some suitable assumptions, all of the four equilibria maybe globally asymptotically stable, that is, the dynamic behaviors of the system becomes complicated with the density dependent birth rate. For example, both species could be driven to extinction or the second species will be driven to extinction while the first species is globally asymptotically stable, as shown in Example 3.1 and 3.2. Such kinds of phenomena are first time observed. We could control the dynamic 
behaviors of the system by adjust the birth rate of the species, and this may be useful in exploit natural resources.

\section{Acknowledgement}

This paper was supported by the Natural Science Foundation of Fujian Eduction Department under Grants (JA12369, JAT160700).

\section{REFERENCES}

[1] A. Xiao, C. Lei, Dynamic behaviors of a non-selective harvesting single species stage-structured system incorporating partial closure for the populations, Adv. Difference Equ. 2018 (2018), Article ID 245.

[2] J.F. Zhang, Bifurcated periodic solutions in an amensalism system with strong generic delay kernel, Math. Methods Appl. Sci. 36 (2013), 113-124.

[3] Q. Lin, Allee effect increasing the final density of the species subject to the Allee effect in a Lotka-Volterra commensal symbiosis model, Adv. Difference Equ. 2018 (2018), Article ID 196.

[4] K. Yang, Z. Miao, F. Chen, X. Xie, Influence of single feedback control variable on an autonomous Holling-II type cooperative system, J. Math. Anal. Appl. 435 (2016), 874-888.

[5] Y. Liu, X. Xie, Q. Lin, Permanence, partial survival, extinction, and global attractivity of a nonautonomous harvesting Lotka-Volterra commensalism model incorporating partial closure for the populations, Adv. Difference Equ. 2018 (2018), Article ID 211.

[6] C. Lei, Dynamic behaviors of a stage structure amensalism system with a cover for the first species, Adv. Difference Equ. 2018 (2018), Article ID 272.

[7] Z. Zhu, Q. Chen, Mathematic analysis on ammensalism Lotka-Volterra model of populations, J. Jixi Univ. 8 (2008), 100-101.

[8] Z. Zhang, Stability and bifurcation analysis for a amensalism system with delays, Math. Numer. Sin. 30 (2008), $213-224$.

[9] R. Wu, Dynamic behaviors of a nonlinear amensalism model, Adv. Difference Equ. 2018 (2018), Article ID 187.

[10] B. Chen, Dynamic behaviors of a non-selective harvesting Lotka-Volterra amensalism model incorporating partial closure for the populations, Adv. Difference Equ. 2018 (2018), Article ID 111.

[11] X. Xie, F. Chen, M. He, Dynamic behaviors of two species amensalism model with a cover for the first species, J. Math. Computer Sci. 16 (2016), 395-401.

[12] R. Wu, L. Zhao, Q. Lin, Stability analysis of a two species amensalism model with Holling II functional response and a cover for the first species, J. Nonlinear Funct. Anal. 2016 (2016), Article ID 46.

[13] R. Wu, A two species amensalism model with non-monotonic functional response, Commun. Math. Biol. Neruosci. 2016 (2016), Article ID 19.

[14] F. Brauer, C. Castillo-Chavez, Mathematical Models in Population Biology and Epidemiology, Springer, New York, 2000.

[15] S. Tang, L. Chen, Density-dependent birth rate, birth pulses and their population dynamic consequences, J. Math. Biol. 44 (2002), 185-199.

[16] L. Berezansky, E. Braverman, L. Idels, Nicholsons blowflies differential equations revisited: main results and open problems, Appl. Math. Model. 34 (2010), 1405-1417.

[17] F. Chen, Y. Xue, Q. Lin, X. Xie, Dynamic behaviors of a Lotka-Volterra commensal symbiosis model with density dependent birth rate, Adv. Difference Equ. 2018 (2018), Article ID 296.

[18] L. Chen, Mathematical Models and Methods in Ecology, Science Press, Beijing, 1988. 